\title{
Gene cloning, functional expression and characterization of a novel GH46 chitosanase from Streptomyces avermitilis (SaCsn46A)
}

Jing Guo ( $\sim$ guojing113@cczu.edu.cn )

Changzhou University https://orcid.org/0000-0002-7622-6810

\section{Yi Wang}

Changzhou University

\section{Wenjun Gao}

Changzhou University

\section{Xinrou Wang}

Changzhou University

\section{Xin Gao}

Changzhou University

\section{Zaiwei Man}

Changzhou University

\section{Zhiqiang Cai}

Changzhou University

\section{Qing Qing}

Changzhou University

\section{Research Article}

Keywords: Streptomyces avermitilis, Chitosanase, Glucosamine, Chitooligosaccharides, Glycoside hydrolase (GH) family 46, Enzyme properties

Posted Date: August 11th, 2021

DOl: https://doi.org/10.21203/rs.3.rs-785979/v1

License: (c) (i) This work is licensed under a Creative Commons Attribution 4.0 International License. Read Full License

Version of Record: A version of this preprint was published at Applied Biochemistry and Biotechnology on September 20th, 2021. See the published version at https://doi.org/10.1007/s12010-021-03687-6. 


\section{Abstract}

A novel glycoside hydrolase (GH) family 46 chitosanase (SaCsn46A) from Streptomyces avermitilis was cloned and functionally expressed in Escherichia coli Rosetta (DE3) strains. SaCsn46A consists of 271 amino acids, which includes a 34-amino acids signal peptide. The protein sequence of SaCsn46A shows maximum identity (83.5\%) to chitosanase from Streptomyces $s p$. SirexAA-E. Then the mature enzyme was purified to homogeneity through Ni-chelating affinity chromatography with a recovery yield of $78 \%$ and the molecular mass of purified enzyme was estimated to be $29 \mathrm{kDa}$ by SDS-PAGE. The recombinant enzyme possessed a temperature optimum of $45^{\circ} \mathrm{C}$ and a pH optimum of 6.2 , and it was stable at pH ranging from 4.0 to 9.0 and below $30^{\circ} \mathrm{C}$. The $K_{\mathrm{m}}$ and $V_{\max }$ values of this enzyme were $1.32 \mathrm{mg} \cdot \mathrm{mL}^{-1}$, $526.32 \mu \mathrm{M} \cdot \mathrm{mg}^{-1} \cdot \mathrm{min}^{-1}$, respectively (chitosan as substrate). The enzyme activity can be enhanced by $\mathrm{Mg}^{2+}$ and especially $\mathrm{Mn}^{2+}$, which could enhance the activity about 3.62 -fold at a $3 \mathrm{mM}$ concentration.

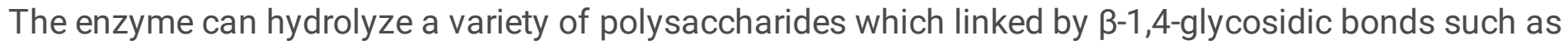
chitin, xylan and cellulose, but it could not hydrolyze polysaccharides linked by a-1,4-glycosidic bonds. The results of thin layer chromatography and HPLC showed that the enzyme exhibited an endo-type cleavage pattern and could hydrolyze chitosan to glucosamine $(\mathrm{GlcN})$ and $(\mathrm{GlcN})_{2}$. This study demonstrated that SaCsn46A is a promising enzyme to produce glucosamine and chitooligosaccharides (COS) from chitosan.

\section{Introduction}

Chitosan is a kind of linear polymer which was composed of $\beta$-1, 4-linked D-glucosamine (GIcN) units. Chitosan is a group of biomacromolecules that is commonly prepared by the partial chemical deacetylation of chitin $(34,4)$. As the second most abundant polymer in nature, chitin occurs in the exoskeletons of fungi, crustaceans, insects and algae. (14).

Chitooligosaccarides (COS) are the hydrolyze products of chitosan with degrees of polymerization (DP) 2-10(26). Cos have many biological activities such as anti-tumor(21), immune enhancing(19), antioxidizing(13), free radical scavenging(15) and antimicrobial activity(33). GlcN has gained its popularity as nutraceutical having application in weight loss and in treatment of osteoarthritis, knee pain and back pain(9). Consequently, it is a popular compound in medicine, agriculture and rises interest in converting chitosan into $\mathrm{COS}$ and $\mathrm{GICN}(24)$. COS and GlcN can be prepared from chitosan by a variety of methods, such as chemical, physical and enzymatic(10). Compare with other methods, enzymatic conversion to produce $\mathrm{COS}$ and GICN produced has attracted extensive attention as an environmentally friendly, high efficiency and controllable and alternative to chemical and physical production. Many enzymes have been reported to hydrolyze chitosan to produce chitooligosaccharides (COS) such as chitosanase, chitinase(22), protease(20), cellulase, lipase(28). Among these enzymes, chitosanase (EC. 3.2.1.132) is the most attractive because of its high activity and specificity.

Chitosanase is a kind of enzyme that hydrolyzes chitosan degradation into COS or GlcN. Various chitosanases have been identified from many species(17). Due to the sequences in the Carbohydrate 
Active Enzymes database (www.cazy.org)(16), chitosanases are categorized into glycoside hydrolases (GH) families $46(24,26,29), 75(34), 80(32)$ and $8(35)$. GH46, GH75, and GH80 currently contain exclusively chitosanases, while the GH8 family contains a few other glycoside hydrolases, such as cellulase, lichenase, and xylanase(35). It is remarkable that chitosanases from fungi are mainly distributed among the $\mathrm{GH} 75$ family, but chitosanases from bacteria are mainly belonging to $\mathrm{GH} 46$ family, a few belong to $\mathrm{GH} 80(17)$.

S. avermitilis is a kind of aerobic bacteria and were mostly reported to produce avermectins(1) and ivermectin(3). Heggset reported a chitosanase from the $\mathrm{GH} 75$ family produced by $S$. avermitilis, which mainly produce long oligomers (DP $\geq 2)(5)$. However, to our knowledge, no report is available regarding on gene cloning and characterization of GH46 family chitosanase from S. avermitilis. Accordingly, in this study, a novel chitosanase designated as SaCsn46A was cloned by PCR and functionally expressed in $E$. coli Rosetta (DE3). The characterization of its biochemical properties suggests that SaCsn46A has high activity and pH stability and could efficiently convert colloidal chitosan into $\mathrm{GlcN}$ and $(\mathrm{GlcN})_{2}$, which was rarely reported by other chitosanases.

\section{Materials And Methods}

\section{Culture medium, chemicals, strains, and plasmids}

Escherichia coli JM 109, E. coli DH5a and E. coli Rosetta (DE3) used in this study was grown at $37^{\circ} \mathrm{C}$ with shaking at 160 r.p.m. in Luria-Bertani medium which contained $\left(g \cdot L^{-1}\right)$ peptone 10 , yeast extract 5 , $\mathrm{NaCl}$ 10. Gene cloning was conducted through the plasmids pMD18-T and E. coli JM109 (Takara). Gene expression was conducted through the plasmids PET-28a (Novagen, Germany) and E. coli Rosetta (Takara). The genetic manipulation reagents (Ex Taq ${ }^{\text {TM }}, T 4$ DNA ligase, restriction endonuclease, buffers and dNTP) were purchased from Takara (Dalian, China). The genomic DNA extraction, plasmid isolation kits, ampicillin, kanamycin, isopropyl $\beta$-D-1-thiogalactopyranoside (IPTG), protein purification and protein electrophoresis reagents were purchased from Sangon (Shanghai, China). COSs with 2-3 DPs were purchased from Qingdao BZ Oligo Biotech Co., Ltd. (Qingdao, China).

\section{DNA cloning and sequence analysis}

Two primers (sacsnF, 5'-3': CGGGATCCGCACCCGTCGGCCTGGACGAC; sacsnR, 5'-3': CCCAAGCTTTCAGCCGATGTGGTAGCTGTC) were designed and used to amplify the chitosanase gene, and the signal peptide was eliminated. The PCR products were cloned into PMD18-T and then digested by the designated restrictive endonuclease and cloned into the corresponding sites (BamHI-HindIII) of the expression vector pET-28a to generate pET-SaCsn46A, the recombinant enzyme carry the His-tag and T7tag belonging to the $\mathrm{pET}-28 \mathrm{a}$ plasmid at the $\mathrm{N}$-terminal. The signal peptide was predicted using Signal $\mathrm{P}$ 4.0 (http://www.cbs.dtu.dk/services/SignalP/). Homology searches in GenBank were performed using the BLAST program. The molecular mass of the protein was determined using the software DNAMAN (http://www.lynnon.com/). Multiple alignments of SaCsn46A sequence were performed with Clustal W 
(https://www.genome.jp/tools-bin/clustalw) and ESprint 3.0 (http://espript.ibcp.fr/ESPript/ESPript/). Phylogenetic trees were constructed using neighbor-joining algorithm in MEGA 5.0 (http://www.megasoftware.net/). Three-dimensional structure homology model of SaCsn46A was constructed by Swiss-Model (http://swissmodel.expasy.org)

\section{Expression and purification of SaCsn46A}

E. coli Rosetta (DE3) cells harboring the designated plasmid were incubated in Luria-Bertani medium containing $50 \mu \mathrm{g} / \mathrm{mL}$ kanamycin and grown at $37^{\circ} \mathrm{C}$ to $\mathrm{OD}_{600}$ of 0.6 . The incubation of the target protein production was performed by adding IPTG to a final concentration of $1 \mathrm{mM}$. The cultures were grown for an additional $12 \mathrm{~h}$ at $16^{\circ} \mathrm{C}$, and protein expression in the cells and culture supernatant were analyzed by SDS-PAGE.

All the following purification steps were carried out at $4{ }^{\circ} \mathrm{C}$. To purify recombinant chitosanase, the induced culture was centrifuged for $10 \mathrm{~min}$ at $10000 \mathrm{~g}$. The cell pellet was suspended in buffer I $(0.02 \mathrm{M}$ Tris- $\mathrm{HCl}, \mathrm{pH}$ 8.0, $0.5 \mathrm{M} \mathrm{NaCl}$ and $10 \%$ glycerol) and washed twice with the same buffer. The washed cell pellet was suspended in buffer I, disrupted by sonication on an ice bath, and then centrifuged for 30 min at $10000 \mathrm{~g}$. The resulting supernatant was applied on a Ni-Sepharose column previously charged with $\mathrm{Ni}$ (II). After the column was washed with buffer II (0.02 M Tris-HCl, pH 8.0, 0.5 M NaCl, 0.02 M imidazole and $10 \%$ glycerol), elution was performed with a $0.08 \mathrm{M}$ imidazole $(0.02 \mathrm{M} \mathrm{Tris-HCl}, \mathrm{pH} 8.0,0.5 \mathrm{M} \mathrm{NaCl}$, $0.08 \mathrm{M}$ imidazole and $10 \%$ glycerol). Elution contains chitosanase activity was collected, imidazole was removed by dialysis, and the enzyme was stored at $-20^{\circ} \mathrm{C}$.

\section{Properties of purified SaCsn46A}

Chitosanase activity was determined by 3, 5-dinitrosalicylic acid (DNS) method, as described previously(4). The content of reducing sugar was determined by measuring optical density (OD) at 520 nm using D-(+)-glucosamine as standard. The reaction mixture was incubated at $45^{\circ} \mathrm{C}$ for $10 \mathrm{~min}$. All reactions were done in triplicate, and their mean and standard deviation values were used for analysis. One unit $(U)$ of chitosanase activity is defined as the amount of enzyme liberating $1 \mu \mathrm{mol}$ of $D-(+)-$ glucosamine-equivalent reducing sugars per minute under the above conditions.

Chitosanase profiles assay for $\mathrm{pH}$ were determined at $\mathrm{pH}$ ranging from 3.0 to 8.0 at $40{ }^{\circ} \mathrm{C}$ in the following buffers: $0.05 \mathrm{M}$ acetate buffer ( $\mathrm{pH}$ 4.5-6.0) and $0.05 \mathrm{M}$ phosphate buffer ( $\mathrm{pH}$ 6.0-8.0). pH stability of chitosanase was determined by measuring the residual activities under optimal conditions $(\mathrm{pH} 6.2,10$ min) after pre-incubation at $0^{\circ} \mathrm{C}$ and $\mathrm{pH} 4.0-9.0$. Buffers used were $0.05 \mathrm{M}$ acetate buffer $(\mathrm{pH} 4.0-6.0)$, $0.05 \mathrm{M}$ phosphate buffer ( $\mathrm{pH}$ 6.0-8.0) and 0.05M Tris-HCl buffer ( $\mathrm{pH}$ 9.0). Chitosanase profiles assay for temperature were determined at temperatures ranging from 20 to $90^{\circ} \mathrm{C}$ at 5 intervals at optimal pH. Thermostability was conducted by measuring the residual activities under optimal conditions $(\mathrm{pH} 6.2,45$ ${ }^{\circ} \mathrm{C}$ ) after pre-incubation at $0-60^{\circ} \mathrm{C}$ and $\mathrm{pH} 7.5$ for $2 \mathrm{~h}$. $\mathrm{pH}$ stability was conducted by measuring the residual activities under optimal conditions after pre-incubation at $0^{\circ} \mathrm{C}$ and $\mathrm{pH} 3.0-9.0$ for $2 \mathrm{~h}$. A control sample with no pretreatment was taken as $100 \%$. 
The substrate specificity of SaCsn46A was determined by DNS method. The reaction mixtures, containing $0.05 \mathrm{M}$ phosphate buffer $(\mathrm{pH} 6.2)$, the tested polysaccharide substrates (1\%) and appropriate amount of SaCsn46A, were incubated at 45 for 20 min. Control samples were treated in the same way in the absence of SaCsn46A. The amount of reducing sugar in the supernatant was determined by measuring the OD520. One unit (U) of enzyme activity was defined as the amount of enzyme that liberated $1 \mu \mathrm{mol}$ of reducing sugar per min under the above assay conditions.

The effects of metal ions on chitosanase activity were determined by assaying the enzyme activity at $\mathrm{pH}$ 6.2 and $45^{\circ} \mathrm{C}$ for $10 \mathrm{~min}$ in the presence of various ions at different concentration.

The catalytic constant was determined by Lineweaver-Burk plot method using chitosan in ranging of $0.1-$ $10 \mathrm{mg} / \mathrm{mL}$ at optimal pH 6.2 and $45^{\circ} \mathrm{C}$ for $10 \mathrm{~min}$ in the presence of $3 \mathrm{mM} \mathrm{Mn}^{2+}$.

\section{Hydrolytic properties of purified SaCsn46A}

The analysis of the hydrolytic products of SaCsn46A was performed by analyzing the products of colloidal chitosan $(1 \%, \mathrm{w} / \mathrm{v} ; 90 \%$ DDA) using thin layer chromatography (TLC) method. The purified enzyme was incubated with colloidal chitosan at $28^{\circ} \mathrm{C}$ in phosphate buffer (pH 6.2). Samples withdrawn at different times were immediately boiled for $20 \mathrm{~min}$, then centrifuged for $10 \mathrm{~min}$ at $10000 \mathrm{~g}$. The hydrolysis products were spotted onto a silica gel plate, which was developed in a solvent system which contains ethyl acetate-methanol-water-25\% ammonia (5:9:1:1.5, v/v/v/v). The plate was sprayed with $0.3 \%$ ninhydrin (dissolved in $95 \%$ ethanol, $\mathrm{w} / \mathrm{v}$ ), followed by heating at $120^{\circ} \mathrm{C}$ for $10 \mathrm{~min}$. The hydrolysis products were analyzed by HPLC system (Waters 1525, Waters, USA) equipped with evaporative light scattering detector (ELSD). The $\mathrm{GlcN}$ and $(\mathrm{GlcN})_{2}$ were separated on high performance sugar column (Shodex Asahipak $\mathrm{NH}_{2}$ P504E, Shodex, Kyoto, Japan), eluted by acetonitrile and distilled water (70/30, $\mathrm{w} / \mathrm{w}$ ) mixture with a flow rate of $1.0 \mathrm{~mL} \cdot \mathrm{min}^{-1}$ at $30^{\circ} \mathrm{C}$.

\section{Results And Discussion}

\section{Sequence analysis of the chitosanase}

Based on Genbank database and amino acid sequence analysis, the chitosanase gene from $S$. avermitilis (GenBank: KUN55720.1) was selected for analysis. The gene contained 816 bp (base pair), which encoding 271 amino acids. The SaCsn46A has a 'leader peptide' in the secretion of this enzyme and a cleavage site at the N-terminus (Ala34-Ala35) which was determined by the Signal P 4.0 program. The protein sequence of SaCsn46A shows maximum identity to chitosanase from Streptomyces sp. (Genbank accession No. WP_028800608.1).

The protein sequences of SaCsn46A and other chitosanases belonging to four different GH families were selected from the GenBank database to constructed a phylogenetic tree (see Fig. 1(a)). SaCsn46A is clearly a member of $\mathrm{GH} 46$ family chitosanase. As can be seen from the multiple sequence alignment of GH 46 family members in Fig. 1(b), the SaCsn46A contained two key active site residues (E22 and D40), 
that are conserved in all GH46 family members. The residues E22 and D40 were replaced by A22 and A40, respectively and the mutants were mostly inactive (data not shown).

Homology model of SaCsn46A was built by the Swiss-Model server with the chitosanase from Streptomyces sp. SirexAA-E as template (PDB ID: 4ily.1A), which shares $83.5 \%$ identity to SaCsn46A. Two amino acid residues in the catalytic active center (E22 and D40) were illustrated with sticks in the 3Dstructure of SaCsn46A. These results suggest that SaCsn46A is a novel member of family GH46.

\section{Heterologous expression and purification of the chitosanase}

The SaCsn46A gene was cloned from S. avermitilis and expressed in E. coli Rosetta (DE3) successfully with a N-terminal His-tag and without signal peptide. The recombinant enzyme were purified to electrophoretic homogeneity by a Ni-NTA affinity column (result was shown in Fig. S1). The molecular weight of purified recombinant enzyme was estimated to be $29 \mathrm{kDa}$ by SDS-PAGE which was about 4 $\mathrm{kDa}$ larger than the wild mature enzyme, corresponding to the size of T7-tag and His-tag at N-terminal of the recombinant enzyme ( $3.7 \mathrm{kDa})$. The molecular weight of the most reported chitosanases were ranging from 20 to $60 \mathrm{kDa}(31,23,7,6,24)$, but Kim reported a chitosanase produced by Aspergillus fumigatus was $108 \mathrm{kDa}(8)$ and Chen obtained a chitosanase produced by Aspergillus sp. CJ22-326 was $109 \mathrm{kDa}(2)$. The molecular weight of SaCsn46A is close to a chitosanase from Streptomyces albolongus $(29.6 \mathrm{kDa})(4)$.

\section{Chitosanase activity and substrate specificity}

The specific activity of SaCsn46A was determined after purification step with Ni-NTA column was increased from $121.2 \mathrm{U} \cdot \mathrm{mg}^{-1}$ to $424.2 \mathrm{U} \cdot \mathrm{mg}^{-1}$, obtaining a purification fold of 3.5 and recovery yield of 78\% (Results are listed in Table 1). 
Table 1

Effects of different subtracts on SaCsn46A

\begin{tabular}{|ll|}
\hline Subtracts & Relative activity (\%) \\
\hline Colloidal chitosan & $100 \pm 1.67$ \\
\hline Power chitosan & $1.77 \pm 0.12$ \\
\hline Colloidal chitin & $1.77 \pm 0.22$ \\
\hline Power chitin & $1.48 \pm 0.17$ \\
\hline Cellulose & $0.46 \pm 0.09$ \\
\hline Carboxymethyl cellulose & $2.39 \pm 0.15$ \\
\hline Xylan & $2.23 \pm 0.17$ \\
\hline Soluble starch & N.D. \\
\hline Dextrin & N.D. \\
\hline N.D., the enzyme activity & was not detected. \\
\hline
\end{tabular}

In order to determine the substrate specificity of SaCsn46A, we tested its ability to hydrolyze different polysaccharides, and the results are listed in Table 2. We found that SaCsn46A can hydrolyze a variety of

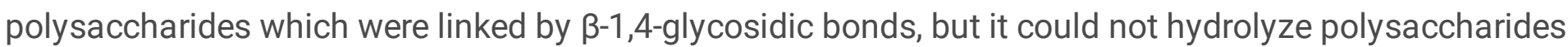
linked by a-1,4-glycosidic bonds such as soluble starch and dextrin. The enzyme showed a high hydrolysis activity toward colloidal chitosan, and displayed low hydrolysis activity toward powder chitosan $(7.51 \pm 0.72 \mathrm{U} / \mathrm{mg})$, colloidal chitin $(7.51 \pm 0.93 \mathrm{U} / \mathrm{mg})$, powder chitin $(6.28 \pm 0.93 \mathrm{U} / \mathrm{mg})$, cellulose $(1.95 \pm 0.93 \mathrm{U} / \mathrm{mg})$, carboxymethyl cellulose $(10.14 \pm 0.64 \mathrm{U} / \mathrm{mg})$ and xylan $(9.46 \pm 0.93 \mathrm{U} / \mathrm{mg})$, shows a broad of substrate spectrum. It can be seen from the experimental results that although SaCsn46A can hydrolyze a variety of polysaccharides, its catalytic capacity for cellulose is the lowest, which may be related to the solubility of cellulose and the space size of constituent monomers. The broad spectrum properties of substrate are different from the reported chitosanase belonging to family GH46, such as Csn21c from S. albolongus(4), Csn-BAC from Bacillus sp. MD-5(29), Csn-CAP from Staphyloccus capitis(24) and BaCsn46A from Bacillus amyloliquefaciens(18). These enzymes strictly hydrolyze colloidal chitosan and have no catalytic effect on powder chitosan and other polysaccharides. 
Table 2

Effects of different ions on SaCsn46A

\begin{tabular}{|lll|}
\hline Ions & \multicolumn{2}{l|}{ Relative activity (\%) } \\
\hline & $1 \mathrm{mM}$ & $2 \mathrm{mM}$ \\
\hline $\mathrm{Control}$ & $100 \pm 1.89$ & $100 \pm 2.18$ \\
\hline $\mathrm{Cu}^{2+}$ & $95.32 \pm 2.45$ & $46.76 \pm 2.34$ \\
\hline $\mathrm{Fe}^{2+}$ & $103.20 \pm 2.04$ & $107.25 \pm 1.51$ \\
\hline $\mathrm{K}^{+}$ & $100.67 \pm 1.88$ & $79.92 \pm 2.52$ \\
\hline $\mathrm{Mg}^{2+}$ & $103.73 \pm 1.98$ & $127.85 \pm 1.74$ \\
\hline $\mathrm{Ba}^{2+}$ & $92.79 \pm 1.94$ & $76.94 \pm 2.43$ \\
\hline $\mathrm{Ca}^{2+}$ & $87.85 \pm 1.59$ & $63.47 \pm 1.89$ \\
\hline $\mathrm{Zn}^{2+}$ & $89.05 \pm 2.62$ & $48.70 \pm 2.09$ \\
\hline $\mathrm{EDTA}^{2+}$ & $34.56 \pm 3.28$ & $21.61 \pm 2.79$ \\
\hline
\end{tabular}

\section{Biochemical characterization of the purified chitosanase}

The optimal temperature for SaCsn46A was determined in different temperature ranges $\left(20-90^{\circ} \mathrm{C}\right)$. The maximum relative chitosanase activity was observed at $45^{\circ} \mathrm{C}$ (Fig. $3 \mathrm{a}$ ), similar optimal temperature (45 ${ }^{\circ} \mathrm{C}$ ) has been reported from Paenibacillus dendritiformis chitosanase(25), while was higher than that Gynuella sunshinyii chitosanase $\left(30^{\circ} \mathrm{C}\right)(17)$, Bacillus sp. BY01 chitosanase $\left(35^{\circ} \mathrm{C}\right)(30)$, Bacillus sp. MD5 chitosanase $\left(40^{\circ} \mathrm{C}\right)(29)$ and $S$. capitis chitosanase $\left(40^{\circ} \mathrm{C}\right)(24)$, and lower than Bacillus sp. chitosanase $\left(60^{\circ} \mathrm{C}\right)(13), B$. amyloliquefaciens chitosanase $\left(55^{\circ} \mathrm{C}\right)(12)$ and $S$. albolongus chitosanase $\left(50^{\circ} \mathrm{C}\right)(4)$. Most reported microbial chitosanases have an optimum reaction $\mathrm{pH}$ in the range 4 to 8 (27). Optimal chitosanase activity was observed at pH 6.2 in $50 \mathrm{mM}$ phosphate buffer (Fig. 3b), while SaScn46A was $\mathrm{pH}$-sensitive (Fig. 3b), less than 30\% relative activity was observed at $\mathrm{pH}$ value below 6 or above 7, however more than $80 \%$ relative activity was observed at pH6-7 in $50 \mathrm{mM}$ phosphate buffer.

Thermostability illustrated that the SaCsn46A was stable below $30^{\circ} \mathrm{C}$ (Fig. 3c). SaCsn46A has good pH stability at $4.0-9.0$ (Fig. 1d), retained more than $80 \%$ of its maximal activity at pH $5.0-9.0$ after incubation for $2 \mathrm{~h}$, the stability characteristic of SaCsn46A was similar to Bacillus sp. chitosanase (stable at $\mathrm{pH} 3.6-9.8$ below $\left.30^{\circ} \mathrm{C}\right)(13)$.

The effects of metal ions on the activity of SaCsn46A are presented in Table 3. The enzyme activity was inhibited by $\mathrm{Cu}^{2+}, \mathrm{Ba}^{2+}, \mathrm{Ca}^{2+}$ and $\mathrm{Zn}^{2+}$ at both $1 \mathrm{mM}$ and $2 \mathrm{mM}$ concentration. The enzyme activity was inhibited by $\mathrm{K}^{+}$at $2 \mathrm{mM}(79.92 \%) . \mathrm{K}^{+}$and $\mathrm{Mg}^{2+}$ of $1 \mathrm{mM}$ has no effect on enzyme activity. $\mathrm{Fe}^{2+}$ at both 1 $\mathrm{mM}$ and $2 \mathrm{mM}$ displayed slight promoting effect on enzyme activity. Notably, the enzyme activity can be 
increased to 3.62 -fold by $\mathrm{Mn}^{2+}$ at $3 \mathrm{mM}$ concentration, and stimulatory effect was observed when the concentration of $\mathrm{Mn}^{2+}$ was $9 \mathrm{mM}$ (Fig. 4). Most chitosanases can be enhanced activity by $\mathrm{Mn}^{2+}$, for example, Csn21c from S. albolongus(4), Csn-BAC from Bacillus sp. MD-5(29) and CsnB from Bacillus sp. BY01(30), that were enhanced to 2.0-fold, 1.79-fold and 2.57-fold, respectively, after the addition of $\mathrm{Mn}^{2+}$, obviously $\mathrm{Mn}^{2+}$ shows more stimulatory effect on SaCsn46A than other chitosanases. EDTA showed a significant inhibitory effect on SaCsn46A, because EDTA is a chelator of divalent cations, suggesting that this chitosanase is a metalloenzyme.

The $K_{\mathrm{m}}$ value (colloidal chitosan as substrate) of SaCsn46A was $1.32 \mathrm{mg} \bullet \mathrm{mL}^{-1}$, which was lower than that of Csn21c from S. albolongus $\left(7.4 \mathrm{mg} \cdot \mathrm{mL}^{-1}\right)(4)$, BaCsn46A from B. amyloliquefaciens $\left(2.8 \mathrm{mg} \cdot \mathrm{mL}^{-}\right.$ $\left.{ }^{1}\right)(18)$, GsCsn46A from $G$. sunshinyii $\left(1.97 \mathrm{mg} \cdot \mathrm{mL}^{-1}\right)(17)$. Notably, this value is very low, indicating a high affinity with the substrate. The $V_{\max }$ value of SaCsn46A was determined that was $526.32 \mu \mathrm{M} \bullet \mathrm{mg}^{-}$

${ }^{1} \cdot \mathrm{min}^{-1}$, which was higher than that of Csn21c from S. albolongus $\left(263.1 \mu \mathrm{M} \cdot \mathrm{mg}^{-1} \cdot \mathrm{min}^{-1}\right)$ and GsCsn46A from G. sunshinyii $\left(358.65 \mu \mathrm{M} \cdot \mathrm{mg}^{-1} \cdot \mathrm{min}^{-1}\right)(17)$, while was much lower than BaCsn46A from B. amyloliquefaciens $\left(7142.9 \mu \mathrm{M} \cdot \mathrm{mg}^{-1} \cdot \mathrm{min}^{-1}\right)(18)$.

\section{Hydrolytic properties of chitosanase}

The hydrolysis products of colloidal chitosan $(1 \%, \mathrm{w} / \mathrm{v}, 90 \% \mathrm{DDA})$ were detected by TLC, results are listed in Fig. 5. After $7 \mathrm{~h}$ incubation, chitosan was complete degraded by SaCsn46A, two clear spots could be detected on the TLC plate. The mobility ratio of these spots was in good agreement with GlcN and $(\mathrm{GlcN})_{2}$ markers, indicated that this enzyme can hydrolyze chitosan into $\mathrm{GlcN}$ and $(\mathrm{GlcN})_{2}$. This conclusion was followed verified by high performance liquid chromatography (HPLC) analysis (Fig. 4). There is one product peak with an appearance time of $6.192 \mathrm{~min}$ in Fig. 4 (c), which is consistent with the appearance time of GIcN Fig. 4 (a), and the appearance time of the other product peak is 7.245 min, which is consistent with the peak time of (GlcN)2 Fig. 4 (b). The ratio of the two hydrolytic products is 1:2.8 (GICN:(GlcN) $)_{2}$ ). As shown in TLC analysis (Fig. 4), CHOS with different DPs appeared after degraded for $5 \mathrm{~min}$. After $30 \mathrm{~min}$ incubation, $(\mathrm{GlcN})_{2}$ and $(\mathrm{GlcN})_{3}$ became main products. As the reaction continued, only two visible spots were left on the TLC plate after complete degradation. Recently, most microorganisms produced chitosanases were reported to produce DP2-7 CHOS $(11-13,16-18,24,25,29$, 30 ), only Csn21c from S. albolongus was reported that can hydrolyze chitosan into GlcN and $(\mathrm{GlcN})_{2}(4)$, however, the hydrolysis behavior of SaCsn46A is completely different from that of Csn21c. When Csn21c hydrolyzes chitosan, GlcN was existed at the initial stage of the hydrolysis process, however, SaCsn46A hydrolyzes chitosan, higher DP products appear first, then gradually degrade into $\mathrm{GlcN}$ and $(\mathrm{GlcN})_{2}$, that

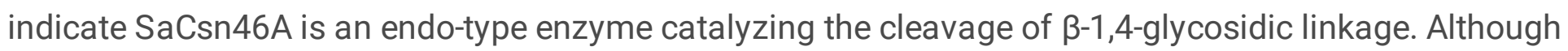
this hydrolysis characteristic of SaCsn46A is similar to other reported GH46 family chitosanases, hydrolysates of SaCsn46A have lower degree of polymerization.

\section{Conclusion}


A novel chitosanase gene was cloned from S. avermitilis and expressed in E. coli Rosetta. The recombinant enzyme has its optimal enzyme activity under condition of $45^{\circ} \mathrm{C}$ in $50 \mathrm{mM} \mathrm{pH} 6.2$ phosphate buffer, and was very stable at $\mathrm{pH}$ range from 4.0 to 9.0 and below $30^{\circ} \mathrm{C}$. The enzyme activity can be strongly enhanced about 3.5-fold by $\mathrm{Mn}^{2+}$ at a $3 \mathrm{mM}$ concentration. The enzyme can hydrolyze a variety of $\beta-1,4$-glycosidic bonds-linked polysaccharides which shows a good application prospect. The hydrolysis products were mainly $\mathrm{GlCN}$ and $(\mathrm{GlcN})_{2}$ which demonstrated that SaCsn46A was a good potential candidate for the industrial production of $\mathrm{GlcN}$ and $(\mathrm{GlcN})_{2}$ in mild conditions.

\section{Declarations}

Author Contribution Conceptualization: J. Guo, Z.W. Man. Methodology: J. Guo, W.J. Gao. Data analysis: J. Guo, Y. Wang. Software: J. Guo. Writing original manuscript: J. Guo. Review and revising manuscript: Y. Wang, W.J. Gao., X.R. Wang, X. Gao, Z.W. Man, Z.Q. Cai, Q. Qing. Funding acquisition: J. Guo, Z.W. Man, Z.Q. Cai, Q. Qing. All authors reviewed and approved the final manuscript.

Funding This work was supported by the National Natural Science Foundation of China (31700075), the Natural Science Foundation of the Jiangsu Higher Education Institution of China (19KJB180001), the Key Research and Development Program of Shandong Province, China (2019JZZY020605), the Initial Research Funding of Changzhou University (ZMF17020115), the Extracurricular Innovation and Entrepreneurship Fund for College Students of Changzhou University (ZMF19020280), the Natural Science Foundation of Jiangsu Province (BK20181465).

Data Availability All data generated or analyzed during this study are included in this published article and its supplementary information files.

Ethics Approval and Consent to Participate Not applicable.

Consent for Publication Not applicable.

Conflict of Interest The authors declare no competing interests.

\section{References}

1. Chen, J., Liu, M., Liu, X., Miao, J., Fu, C., Gao, H. ... Zhang, L. (2016). Interrogation of Streptomyces avermitilis for efficient production of avermectins. Synthetic and Systems Biotechnology, 1, 7-16

2. Chen, X., Xia, W., \& Yu, X. (2005). Purification and characterization of two types of chitosanase from Aspergillus sp. CJ22-326. Food Research International, 38, 315-322

3. Deng, Q., Xiao, L., Liu, Y., Zhang, L., Deng, Z., \& Zhao, C. (2019). Streptomyces avermitilis industrial strain as cell factory for Ivermectin B1a production. Synthetic and Systems Biotechnology, 4, 34-39

4. Guo, N., Sun, J., Wang, W., Gao, L., Liu, J., Liu, Z. ... Mao, X. (2019). Cloning, expression and characterization of a novel chitosanase from Streptomyces albolongus ATCC 27414. Food 
Chemistry, 286, 696-702

5. Heggset, E. B., Tuveng, T. R., Hoell, I. A., Liu, Z., Eijsink, V. G. H., \& Vårum, K. M. (2012). Mode of Action of a Family 75 Chitosanase from Streptomyces avermitilis. Biomacromolecules, 13, 1733-1741

6. Hirano, K., Arayaveerasid, S., Seki, K., Adams, D. J., \& Mitsutomi, M. (2014). Characterization of a Chitosanase from Aspergillus fumigatus ATCC13073. Bioscience, Biotechnology, and Biochemistry, 76, 1523-1528.

7. Kim, P. I., Kang, T. H., Chung, K. J., Kim, I. S., \& Chung, K. (2004). Purification of a constitutive chitosanase produced by Bacillus sp. MET 1299 with cloning and expression of the gene. FEMS Microbiology Letters, 240, 31-39

8. Kim, S. Y. S., \& Lee, D. H., Ke Ho. (1998). Purification and Characteristics of Two Types of Chitosanases from Aspergillus fumigatus KH-94. Journal of Microbiology and Biotechnology, 8, 568-574

9. Kumar, M., Dangayach, P., \& Pareek, N. (2020). Enhanced glucosamine production through synergistic action of Aspergillus terreus chitozymes. Journal of Cleaner Production, 262, 121363

10. Lin, S., Qin, Z., Chen, Q., Fan, L., Zhou, J., \& Zhao, L. (2019). Efficient Immobilization of Bacterial GH Family 46 Chitosanase by Carbohydrate-Binding Module Fusion for the Controllable Preparation of Chitooligosaccharides. Journal of Agricultural and Food Chemistry, 67, 6847-6855

11. Liu, G. L., Li, Y., Zhou, H. X., Chi, Z. M., \& Madzak, C. (2012). Over-expression of a bacterial chitosanase gene in Yarrowia lipolytica and chitosan hydrolysis by the recombinant chitosanase. Journal of Molecular Catalysis B: Enzymatic, 83, 100-107

12. Luo, S., Qin, Z., Chen, Q., Fan, L., Jiang, L., \& Zhao, L. (2020). High level production of a Bacillus amlyoliquefaciens chitosanase in Pichia pastoris suitable for chitooligosaccharides preparation. International Journal of Biological Macromolecules, 149, 1034-1041

13. Ma, C., Li, X., Yang, K., \& Li, S. (2020). Characterization of a New Chitosanase from a Marine Bacillus sp. and the Anti-Oxidant Activity of Its Hydrolysate. Marine Drugs, 18.

14. Mao, X., Guo, N., Sun, J., \& Xue, C. (2017). Comprehensive utilization of shrimp waste based on biotechnological methods: A review. Journal of Cleaner Production, 143, 814-823

15. Park, P. J., Je, J. Y., \& Kim, S. K. (2003). Free Radical Scavenging Activity of Chitooligosaccharides by Electron Spin Resonance Spectrometry. Journal of Agricultural and Food Chemistry, 51, 4624-4627

16. Pechsrichuang, P., Lorentzen, S. B., Aam, B. B., Tuveng, T. R., Hamre, A. G., Eijsink, V. G. H., \& Yamabhai, M. (2018). Bioconversion of chitosan into chito-oligosaccharides (CHOS) using family 46 chitosanase from Bacillus subtilis (BsCsn46A). Carbohydrate Polymers, 186, 420-428

17. Qin, Z., Chen, Q., Lin, S., Luo, S., Qiu, Y., \& Zhao, L. (2018). Expression and characterization of a novel cold-adapted chitosanase suitable for chitooligosaccharides controllable preparation. Food Chemistry, 253, 139-147

18. Qin, Z., Luo, S., Li, Y., Chen, Q., Qiu, Y., Zhao, L. ... Zhou, J. (2018). Biochemical properties of a novel chitosanase from Bacillus amyloliquefaciens and its use in membrane reactor (97 vol., pp. 9-16). LWT 
19. Rahimnejad, S., Yuan, X., Wang, L., Lu, K., Song, K., \& Zhang, C. (2018). Chitooligosaccharide supplementation in low-fish meal diets for Pacific white shrimp (Litopenaeus vannamei): Effects on growth, innate immunity, gut histology, and immune-related genes expression. Fish \& Shellfish Immunology, 80, 405-415

20. Ribas Fonseca, L., Porto Santos, T., Czaikoski, A., \& Cunha, L., R. (2020). Modulating properties of polysaccharides nanocomplexes from enzymatic hydrolysis of chitosan. Food Research International, 137, 109642

21. Salah, R., Michaud, P., Mati, F., Harrat, Z., Lounici, H., Abdi, N. ... Mameri, N. (2013). Anticancer activity of chemically prepared shrimp low molecular weight chitin evaluation with the human monocyte leukaemia cell line, THP-1. International Journal of Biological Macromolecules, 52, 333-339

22. Shehata, A. N., Aty, A. E., Darwish, A. A., Wahab, D. A. A., \& Mostafa, F. A., W. A. and. (2018). Purification, physicochemical and thermodynamic studies of antifungal chitinase with production of bioactive chitosan-oligosaccharide from newly isolated Aspergillus griseoaurantiacus KX010988. International Journal of Biological Macromolecules, 107, 990-999

23. Su, C., Wang, D., Yao, L., \& Yu, Z. (2006). Purification, Characterization, and Gene Cloning of a Chitosanase from Bacillus Species Strain S65. Journal of Agricultural and Food Chemistry, 54, 4208-4214

24. Sun, H., Cao, R., Li, L., Zhao, L., \& Liu, Q. (2018). Cloning, purification and characterization of a novel GH46 family chitosanase, Csn-CAP, from Staphylococcus capitis. Process Biochemistry, 75, 146-151

25. Sun, H., Mao, X., Guo, N., Zhao, L., Cao, R., \& Liu, Q. (2018). Discovery and Characterization of a Novel Chitosanase from Paenibacillus dendritiformis by Phylogeny-Based Enzymatic Product Specificity Prediction. Journal of Agricultural and Food Chemistry, 66, 4645-4651

26. Sun, H., Yang, G., Cao, R., Mao, X., \& Liu, Q. (2020). Expression and characterization of a novel glycoside hydrolase family 46 chitosanase identified from marine mud metagenome. International Journal of Biological Macromolecules, 159, 904-910

27. Thadathil, N., \& Velappan, S. P. (2014). Recent developments in chitosanase research and its biotechnological applications: A review. Food Chemistry, 150, 392-399

28. Xia, W., Liu, P., \& Liu, J. (2008). Advance in chitosan hydrolysis by non-specific cellulases. Bioresource Technology, 99, 6751-6762

29. Yang, G., Sun, H., Cao, R., Liu, Q., \& Mao, X. (2020). Characterization of a novel glycoside hydrolase family 46 chitosanase, Csn-BAC, from Bacillus sp. MD-5. International Journal of Biological Macromolecules, 146, 518-523

30. Yang, Y., Zheng, Z., Xiao, Y., Zhang, J., Zhou, Y., Li, X. ... Yu, H. (2019). Cloning and Characterization of a Cold-adapted Chitosanase from Marine Bacterium Bacillus sp. BY01. Molecules, 24.

31. Yoon, H. G., Kim, H. Y., Lim, Y. H., Kim, H. K., Shin, D. H., Hong, B. S., \& Cho, H. Y. (2000). Thermostable Chitosanase from Bacillus sp. Strain CK4: Cloning and Expression of the Gene and Characterization of the Enzyme. Applied and Environmental Microbiology, 66, 3727 
32. Yorinaga, Y., Kumasaka, T., Yamamoto, M., Hamada, K., \& Kawamukai, M. (2017). Crystal structure of a family 80 chitosanase from Mitsuaria chitosanitabida. FEBS Letters, 591, 540-547

33. Yue, L., Li, J., Chen, W., Liu, X., Jiang, Q., \& Xia, W. (2017). Geraniol grafted chitosan oligosaccharide as a potential antibacterial agent. Carbohydrate Polymers, 176, 356-364

34. Zhang, J., Cao, H., Li, S., Zhao, Y., Wang, W., Xu, Q. ... Yin, H. (2015). Characterization of a new family 75 chitosanase from Aspergillus sp. W-2. International Journal of Biological Macromolecules, 81, 362-369

35. Zhou, Z., Zhao, S., Liu, Y., Chang, Z., Ma, Y., Li, J., \& Song, J. (2016). A highly Conserved Aspartic Acid Residue of the Chitosanase from Bacillus Sp. TS Is Involved in the Substrate Binding. Applied Biochemistry and Biotechnology, 180, 1167-1179

\section{Figures}

\section{Figure 1}

Bioinformatics analysis of SaCsn46A. (a) Neighbor-joining phylogenetic tree. Phylogenetic analysis was carried out using MEGA 5.0 software. Chitosanases in different families are framed in red; (b) multiple amino acid sequence alignment of SaCsn46A and other chitosanases belonging to $\mathrm{GH} 46$ family (signal peptide were removed from these protein sequences). The typical catalytic residues ( $E$ and $D)$ are indicated by red underlines. The listed sequence included the chitosanases from Streptomyces sp. SirexAA-E (4ILY), Streptomyces sp. N174 (1CHK), Microbacterium sp. OU01 (ABM91442.1), Bacillus amyloliquefaciens (QJW64146.1) and Bacillus sp. MD-5 (ASB61783.1); (c) Homology modeling structure of SaCsn46A. 
(a)

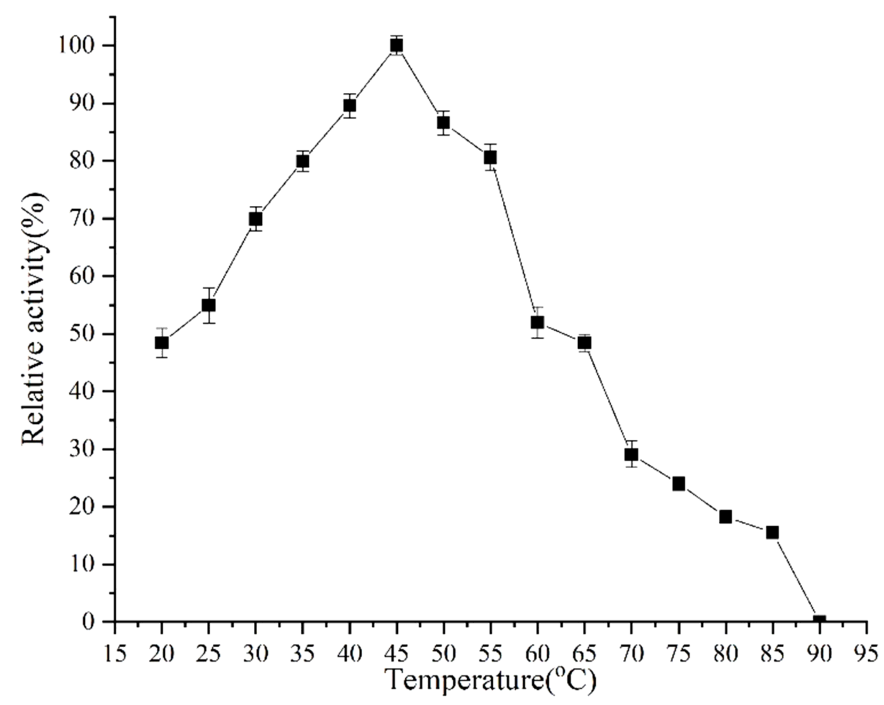

(c)

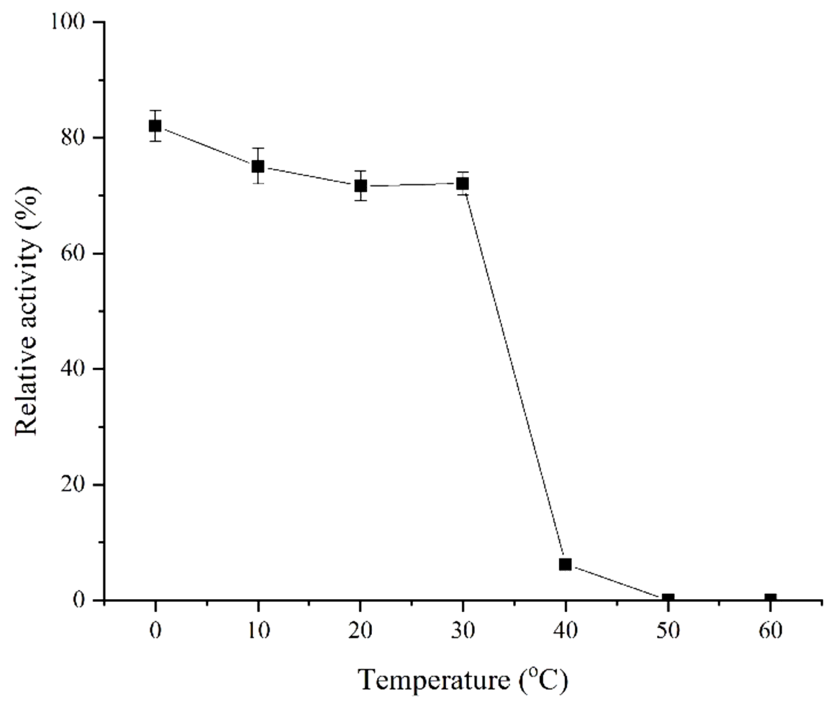

(b)

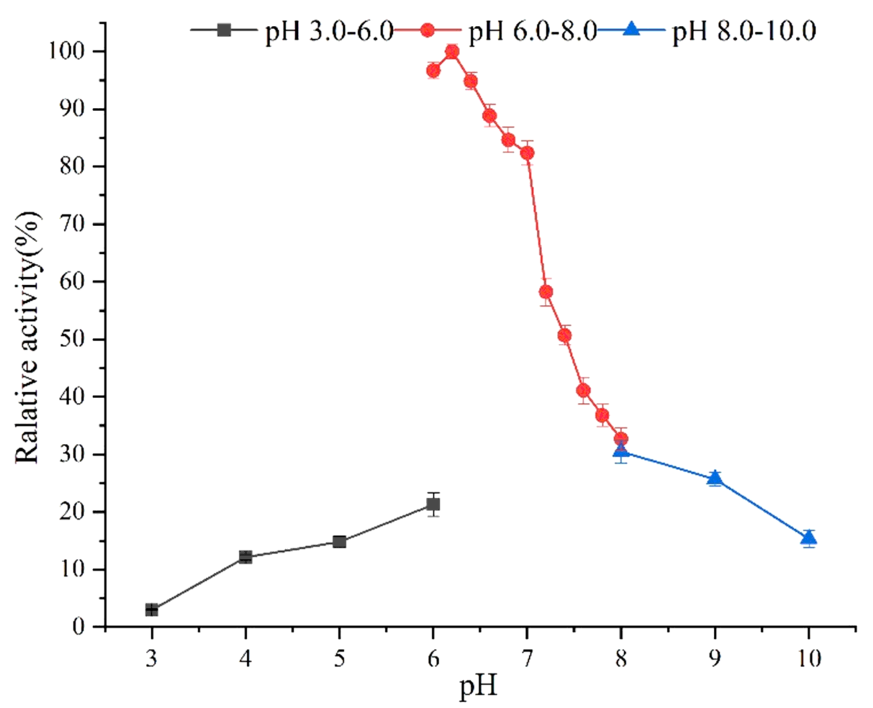

(d)

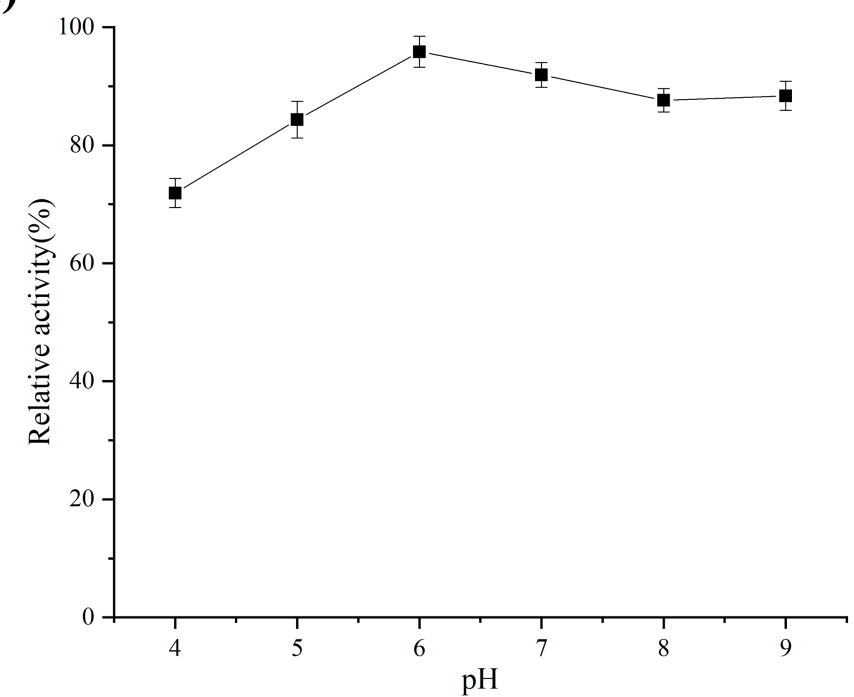

Figure 2

Enzymatic properties of SaCsn46A. (a) Effects of temperature on SaCsn46A; (b) Effects of pH on SaCsn46A; (c) enzyme inactivation at different temperatures; (d) pH stability. The $100 \%$ relative activity of SaCsn46A was $424.2 \mathrm{U} / \mathrm{mg}$. 


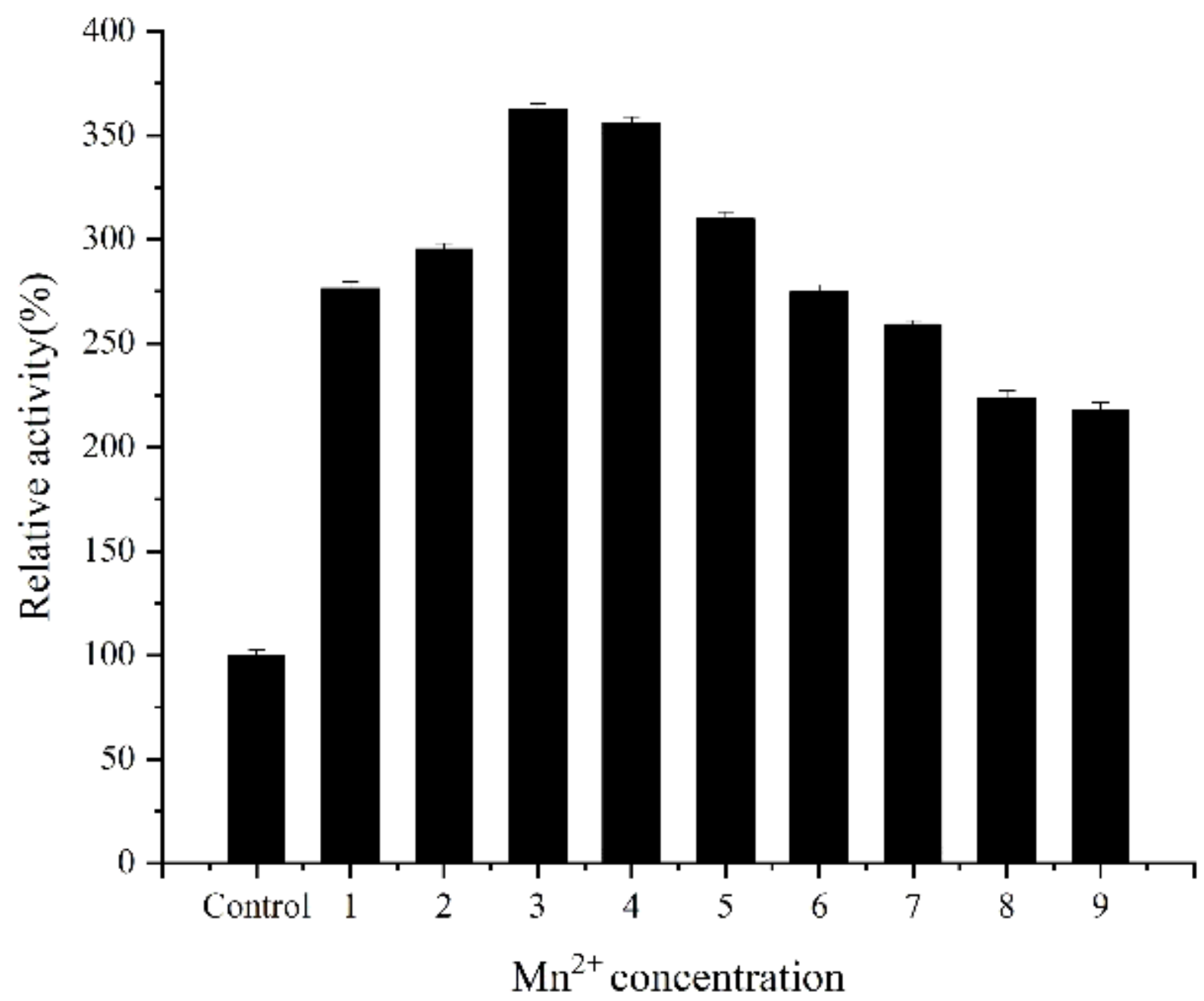

Figure 3

Effects of different Mn2+ concentration on SaCsn46A

Figure 4

Thin layer chromatography (TLC) analysis of hydrolysis products of colloidal chitosan and HPLC analysis of hydrolysis products of colloidal chitosan by SaCsn46A. (a) GIcN standard; (b) (GIcN)2 standard; (c) hydrolysis products of colloidal chitosan by SaCsn46A; Std, COS standards.

\section{Supplementary Files}

This is a list of supplementary files associated with this preprint. Click to download. 
- SupplementalResult.docx

Page 16/16 